\title{
Micro-structured light emission from planar InGaN light-emitting diodes
}

\author{
David Massoubre ${ }^{1,2}$, Enyuan Xie ${ }^{2}$, Benoit Guilhabert ${ }^{2}$, \\ Johannes Herrnsdorf, Erdan Gu, Ian M Watson and Martin D Dawson
}

Institute of Photonics, University of Strathclyde, SUPA, 106 Rottenrow, Glasgow G4 0NW, UK

E-mail: d.massoubre@griffith.edu.au

Received 26 September 2013, in final form 18 October 2013

Published DD MM 2013

Online at stacks.iop.org/SST/28/000000

\begin{abstract}
Investigation of the surface modification of the p-type layer in GaN light-emitting diodes (LEDs) by exposure to a trifluoromethane plasma is reported. It is found that the plasma treatment reduces the conductivity of the $\mathrm{p}-\mathrm{GaN}$ by several orders of magnitude, and when applied at room-temperature through a patterned mask, localized current channels into the active region of a $\mathrm{p}-\mathrm{i}-\mathrm{n}$ device are created. This provides a novel approach to laterally modulate the light emission from an LED over essentially planar areas. This technique allows the projection of high-resolution images from non-pixelated devices, and an example application of maskless pattern transfer with sub-micron features into photoresist is demonstrated.
\end{abstract}

Q1 (Some figures may appear in colour only in the online journal)

\section{Introduction}

During the past decade, much work has been devoted to the development of high power gallium nitride $(\mathrm{GaN})$-based light emitting diodes (LEDs) emitting in the ultraviolet (UV) and visible spectrum. Most of these efforts have concentrated on the improvement of material quality, internal quantum efficiency, and light extraction for general lighting applications [1]. The standard GaN LED fabrication approach is dictated by the use of an insulating substrate material, sapphire. Lightemitting areas are defined as mesas by dry etching of $\mathrm{p}-\mathrm{i}-\mathrm{n}$ epistructures down to the n-layer, resulting in devices with both $\mathrm{n}$ - and $\mathrm{p}$-contacts on the same side of the substrate. While mesas can be as large as a few square millimeters for lighting devices, this same basic approach has been extended to fabricate arrays of micro-pixelated LEDs in which individual pixels may be only a few tens of square micrometers in area. Such micro-pixelated LED arrays are currently the main approach to extend the functionality of GaN-based LEDs to advanced applications including image display [2], visible light communications [3], optical tweezers [4], and maskless

\footnotetext{
${ }^{1}$ Present address: Griffith University, Queensland Micro and Nanotechnology Centre, West Creek Road, Nathan Qld 4111, Australia.

2 These authors have contributed equally to this work.
}

lithography [5]. However, the mesa-etching method of defining pixels has its disadvantages. The introduction of defects during dry etching becomes more problematic as the ratio of sidewall area to pixel area increases [6]. Importantly, the resolution of displayed patterns is limited by the pitch of the pixel array. Furthermore, the non-planar surface left by mesa etching may complicate integration of additional elements in lab-on-chip and related microsystems.

Previous work on ion implantation and plasma treatment of GaN-based structures suggests possible approaches for mesa-free definition of emissive areas in GaN LEDs. Highenergy ion implantation can increase resistivity in both nand p-type nitrides, through introduction of trap states [7]. However, implant isolation has not achieved the same uptake for III-nitride as in other III-V materials, and the damage introduced is an obvious drawback for light-emitting devices. The de-activation of p-type $\mathrm{Mg}$-doped $\mathrm{GaN}$ through the specific mechanism of $\mathrm{Mg}-\mathrm{H}$ complex formation is also well known, and can be achieved through proton implantation [8], simple $\mathrm{H}_{2}$ gas exposure, or plasma treatment [9]. The latter two techniques, however, require additional heating (to at least $300{ }^{\circ} \mathrm{C}$ ) to allow hydrogen to diffuse to a useful depth from the surface, and therefore cannot be used with photoresist (PR) masks for pattern definition. Kuo et al recently reported a method for increasing the contact barrier height to 
p-GaN by exposure to $\mathrm{Cl}_{2} / \mathrm{BCl}_{3} / \mathrm{Ar}$ plasmas, compatible with pattern transfer across a broad-area LED mesa [10]. Finally, $\mathrm{CF}_{4}$-based plasmas have been applied quite extensively to improve the performance of GaN-based high electron mobility transistors (HEMTs) following the initial work of Cai et al [11]. Various effects are believed to contribute, including the implantation of fluorine to depths of $\sim 20 \mathrm{~nm}$ where it can affect the AlGaN-GaN heterojunction present in the HEMT structures.

Here we report a novel technique for defining localized current channels in the $\mathrm{p}$-type layer of $\mathrm{p}-\mathrm{i}-\mathrm{n}$ GaN-based LED structures. This process involves application of a $\mathrm{CHF}_{3}$-based plasma at room temperature, and has two key characteristics allowing light emission across a macroscopic mesa to be spatially modulated in an arbitrary fashion. Firstly, the effect of the plasma treatment is blocked by conventional PR layers, allowing any micron-scale pattern created in the resist to generate an equivalent pattern of current injection into the LED active region. Secondly, the plasma treatment penetrates through a conventional semi-transparent metal layer, which can be applied over the full mesa surface to spread current from a remote p-contact pad. We discuss the scope of this technique for pattern and image display, illustrating the achievable resolution. We also discuss mechanistic aspects of the process, and demonstrate high-fidelity transfer of patterns in maskless lithography.

\section{Experiment}

To demonstrate our novel process, broad emitting-area GaNbased LEDs, with peak emission wavelengths at $405 \mathrm{~nm}$ and $450 \mathrm{~nm}$ respectively, were fabricated following a standard process and using a commercially available $\mathrm{p}-\mathrm{i}-\mathrm{n}$ LED structure grown on a c-plane sapphire wafer. First, the wafer was diced into pieces of appropriate size and cleaned. The samples were next soaked for $3 \mathrm{~min}$ in an $18 \%$ aqueous $\mathrm{HCl}$ solution to remove the native oxide, then rinsed in de-ionized water. This was immediately followed by the deposition with an electron beam evaporator of a bilayer of $\mathrm{Ni} / \mathrm{Au}(15 \mathrm{~nm} / 25 \mathrm{~nm})$ to be used as a current spreading layer. Next, a thin PR layer was spin-coated on each sample and patterned by standard photolithography to define the mesa for each broad emitting-area LED, created by a chlorine-based inductively coupled plasma dry etching step down to the n-doped GaN layer. For a first demonstration, large broadarea mesas with a size of $250 \times 250 \mu \mathrm{m}^{2}$ were defined. The PR pattern remaining after mesa etching was washed off, and the samples underwent rapid thermal annealing at $500^{\circ} \mathrm{C}$ for $120 \mathrm{~s}$ in a purified air ambient to form semi-transparent ohmic contacts to the $\mathrm{p}-\mathrm{GaN}$. This oxidative anneal converts the $\mathrm{Ni}$ metal into a conductive $\mathrm{NiO}_{x}$ phase, that is immiscible with the Au component, resulting in a two-phase nanocomposite [12]. Finally, n-pads and p-pads consisting of a $50 \mathrm{~nm} / 200 \mathrm{~nm}$ $\mathrm{Ti} / \mathrm{Au}$ bilayer were deposited by sputtering and patterned by lift-off processing. In order to create the micro-images, an additional step was added to the standard LED process. This additional step, which can be done at any stage after the p-contact annealing, consists of spin-coating a $500 \mathrm{~nm}$-thick
PR (Microposit S1805, positive tone) layer followed by standard photolithography to transfer a micro-pattern from a photo-mask into the $\mathrm{PR}$ as schematized in figure $1(a)$. Subsequently, the patterned samples underwent a room temperature plasma treatment in a reactive ion etch system (Oxford 80 Plus) using gas flow of 15 standard cubic centimeters per minute ( $\mathrm{sccm}$ ) of $\mathrm{CHF}_{3}$ and $5 \mathrm{sccm}$ of $\mathrm{Ar}$ (the Ar component serves to stabilize the plasma). The plasma power and the chamber pressure were set at $200 \mathrm{~W}$ and $30 \mathrm{~m}$ Torr respectively for a total treatment period of $240 \mathrm{~s}$. The remaining PR mask was then washed off and the plasmatreated devices (named light-structured LEDs in the rest of this paper) were wire bonded onto printed circuit boards for characterization.

Current versus voltage (I-V) characteristics under direct current (dc) drive conditions were measured with a Yokogawa GS610 source measure unit. In order to qualitatively investigate the surface modification effect, metal circular transmission line method (CTLM) semiconductor junctions were processed on the p-doped $\mathrm{GaN}$ surface from the same LED wafer using similar processing as for the LEDs. They consist of co-planar Ti $(40 \mathrm{~nm}) / \mathrm{Au}(150 \mathrm{~nm})$ metal structures comprising circular pads, each with a concentric outer electrode of much larger area, separated by a $10 \mu \mathrm{m}$ gap. Micro-photoluminescence ( $\mu \mathrm{PL})$ spectra were obtained using either a CW GaN-diode laser (PowerTechnologies) with a peak emission at $375 \mathrm{~nm}$ (10 $\mathrm{mW}$ nominal power) or a $\mathrm{CW}$ He-Cd laser (Plasma JSC) with a peak emission at $325 \mathrm{~nm}$ for the excitation source and an UV-visible spectrometer for the detection. The laser beam was focused on the sample with a 60X microscope objective (Nikon, CFI Plan FLuor) giving a spot diameter of $\sim 2 \mu \mathrm{m}$ on the sample. The demonstration of maskless photolithography with LED direct exposure (also called LED direct writing) was made through an imaging setup with 10-to-1 demagnification capability using $4 \mathrm{x}$ and 40x microscope objectives for collection and projection, respectively, as described in previously reported work [5, 13]. The photolithography was performed on a silicon ( $\mathrm{Si}$ ) substrate coated successively with $\mathrm{a} \mathrm{SiO}_{2}$ layer and a layer of negativetone PR (ma-N 1405 from Micro Resist Technology GmbH). The Si sample was placed at the focal plane underneath the projection optics and light emission from a $405 \mathrm{~nm}$ lightstructured LED was used for the demonstration. The exposure dose was controlled by a shutter placed directly in front of the collection optics.

\section{Results and discussion}

\subsection{Device performance and investigations of the $p-G a N$ modification}

Two examples of complex light emission patterns obtained using the process flow described above are presented in figures $1(b)$ and $(c)$. Each complete image is emitted from one LED powered with a single cathode and anode, contrasting with the mode of image generation from pixelated microLED displays $[2,14]$. Each fabricated LED shows a uniform brightness from the non-treated area, and no light emission from the plasma-treated area. The overall size of both 

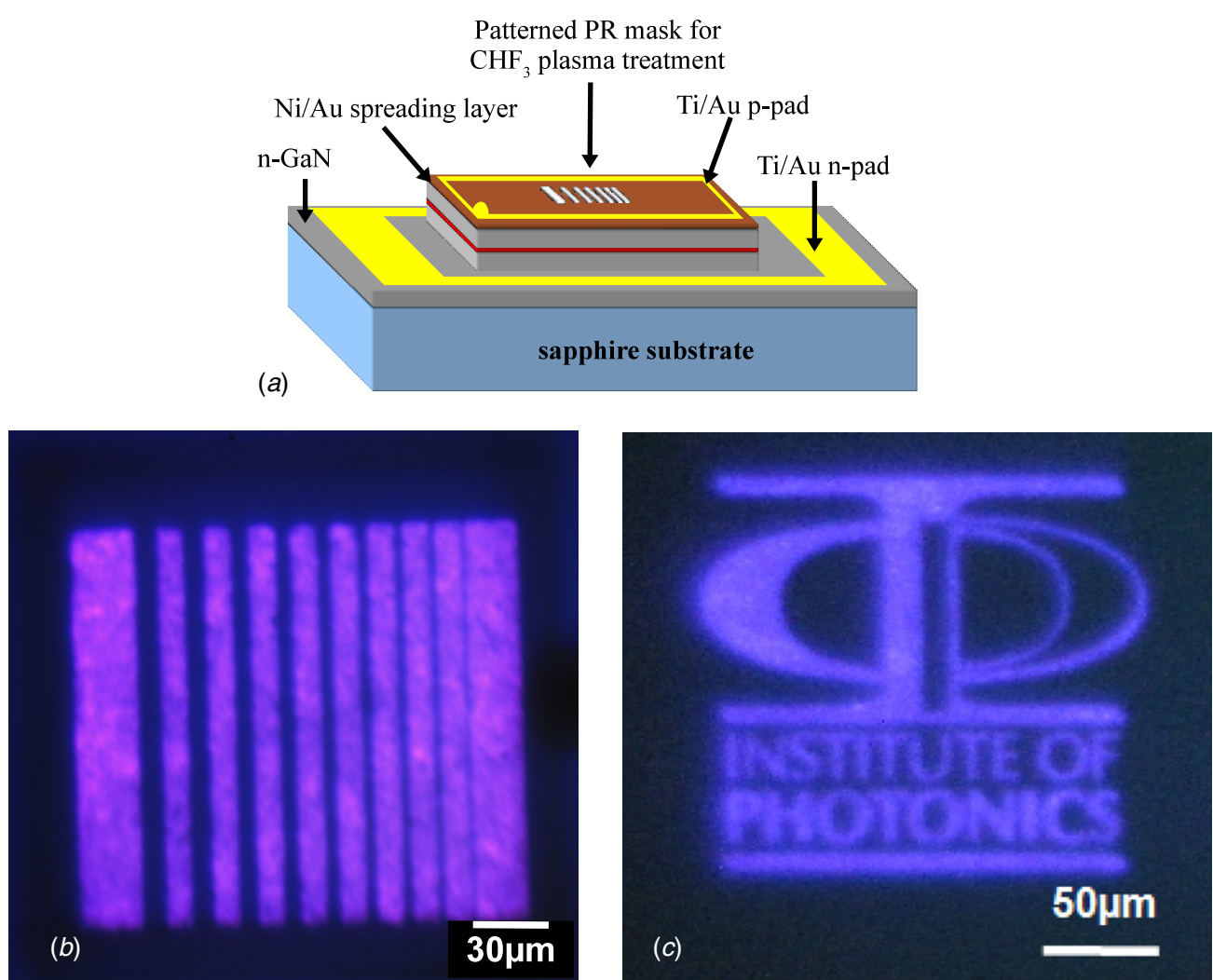

Figure 1. (a) Schematic structure of a LED with the patterned PR mask prior to the $\mathrm{CHF}_{3}$ plasma treatment. (b) Optical micrographs showing a 1D array of bars and $(c)$ the logo of our laboratory displayed respectively by $405 \mathrm{~nm}$ and $450 \mathrm{~nm}$ light-structured turned-on LEDs.

patterns is in the hundreds of micron range, and they show fine features as small as a few microns demonstrating a resolution already much better than the capabilities of pixelated micro-LED displays. Three other initial observations can be made about our process on the basis of the image display results. Firstly, a conventional polymer-based PR layer acts as an efficient mask for the room-temperature plasma treatment, and consequently this process is fully compatible with photolithography techniques used in standard microfabrication. Secondly, the unmasked $\mathrm{NiO}_{x}-\mathrm{Au}$ layer coating the LED emitting surface does not show degradation induced by the plasma treatment and still performs its currentspreading purpose efficiently over the time of this study. Indeed, if the conductance of the current-spreading layer was somehow degraded, either through significant reduction of thickness or alteration of electrical properties, during the process, strong non-uniformity of light emission across the pixel would be expected as well as an important increase of the differential resistance. Further etching tests showed that over the timescale used for the plasma treatment, no measurable etching of the metallic current-spreading layer, nor of the p-GaN layer, could be detected. Finally, one can deduce that as a change in the electrical behavior of the spreading layer cannot explain the localization of light emission, an obvious inference is a strong modification of the electrical properties of the $\mathrm{p}-\mathrm{GaN}$ layer induced by the plasma treatment. As the thin $\mathrm{NiO}_{x}-\mathrm{Au}$ layer is expected to show high porosity after annealing [12], the plasma species can readily interact with the underlying $\mathrm{p}-\mathrm{GaN}$ layer, leading to a spatial modulation of the current injection efficiency, which in turn leads to a strong spatial modulation of the light emission.

Further optical and electrical studies were performed to investigate the effect of the $\mathrm{CHF}_{3}$-based plasma treatment. First, micro-PL measurements were done on treated and untreated areas of a light-structured LED. Excitation at $375 \mathrm{~nm}$ enables direct pumping of the quantum wells (QW) and therefore helps to assess if the plasma treatment causes any damage to the active region, while the $325 \mathrm{~nm}$ source excites mainly the p-type GaN layer. Indeed, $85 \%$ of the $325 \mathrm{~nm}$ pump light will be absorbed within a $200 \mathrm{~nm}$ thick p-type GaN layer and $25 \%$ within the first $30 \mathrm{~nm}$. Results are shown in figure 2. It can be seen that the QW emission obtained under the $375 \mathrm{~nm}$ excitation (figure 2(a)) is basically identical in both treated and untreated regions. As both areas were only a few hundred microns apart for this measurement, the relative peak intensities are directly comparable and consequently it can be concluded that the plasma treatment has no effect on the optical properties of the QWs. Similarly, no clear difference can be noted under the $325 \mathrm{~nm}$ excitation as shown on figure 2(b) with the near band-edge emission (NBE) below $400 \mathrm{~nm}$, the MQW emission centered at $450 \mathrm{~nm}$ and the common GaN's weak yellow luminescence centered at $580 \mathrm{~nm}$ [15]. In particular, the ratio between the NBE and the QWs emission remains constant, which is an indication that the carrier migration from the p-type GaN into the $\mathrm{QW}$ is unaffected by the plasma treatment. This means that either the hole mobility is not affected by the plasma treatment or that it has an effect only in a very thin layer (perhaps $30 \mathrm{~nm}$ or less) at the top of the p-type 

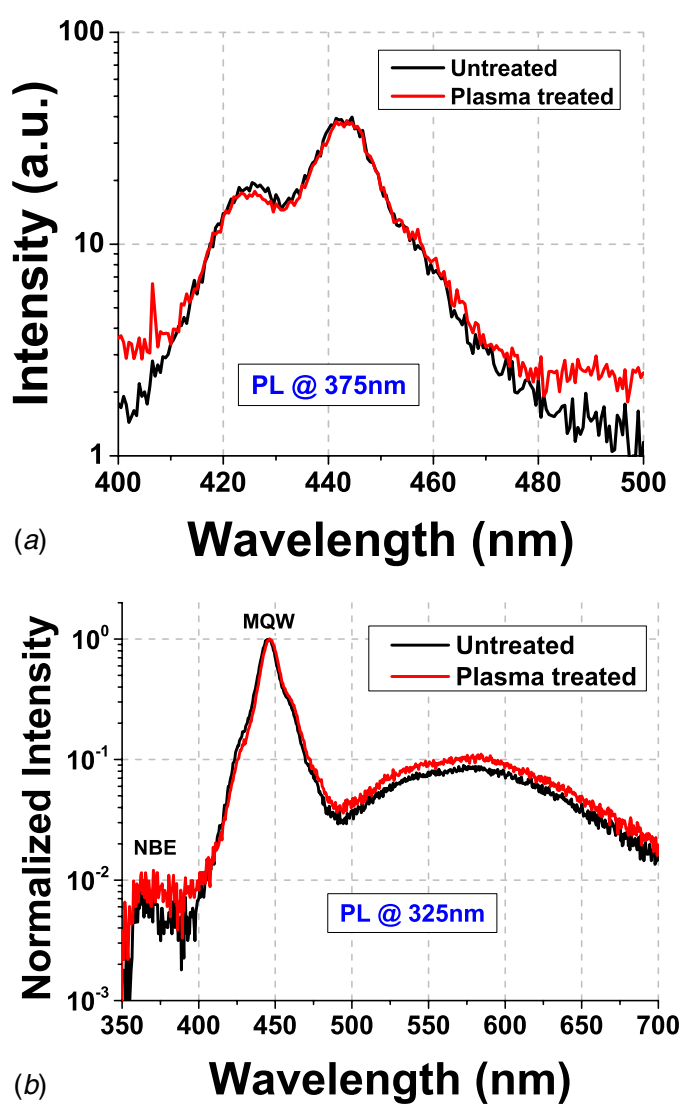

Figure 2. Micro-PL spectra of plasma-treated and untreated regions of a $450 \mathrm{~nm}$ LED excited respectively at $375 \mathrm{~nm}(a)$ and at $325 \mathrm{~nm}(b)$.

GaN layer. This interpretation is in agreement with studies by Chu et al [16] who observed a thin F-implanted surface layer.

For the electrical studies, CTLM structures, resulting in two Schottky metal-semiconductor junctions in series, were made as detailed in the experimental part above. These structures were made on the $\mathrm{p}-\mathrm{GaN}$ surface of samples from the same wafer used to fabricate the $450 \mathrm{~nm}$ light-structured LEDs. Different pre-metallization plasma treatments were tested to elucidate the action of the $\mathrm{CHF}_{3}$ plasma, and the resulting dc I-V characteristics are plotted in figure 3. Comparing the structure exposed to the $\mathrm{CHF}_{3}$ plasma with the nontreated reference sample, a dramatic current reduction, by a factor exceeding $10^{6}$ between $6 \mathrm{~V}$ and $10 \mathrm{~V}$, was observed (comparisons are limited by the noise at lower currents), demonstrating that the $\mathrm{CHF}_{3}$ plasma treatment enables a strong local inhibition of current injection over the whole operating range of standard GaN-based LEDs. Considered together with the PL results, we believe that the change in Schottky barrier behavior after the plasma treatment involves modification of the topmost layer of the p-GaN. Two complementary physical effects may contribute. The first is the well-known de-activation of $\mathrm{Mg}$ acceptors by hydrogen, which results in a decrease of the mobile hole concentration and thus of the p-GaN conductivity [8]. A second effect could be an increase of the effective metal-semiconductor barrier energy due to the implantation of fluorine into the $\mathrm{p}-\mathrm{GaN}$ surface [16]. In order to evaluate these two possible effects in isolation, $\mathrm{H}_{2}$ and $\mathrm{CF}_{4}$

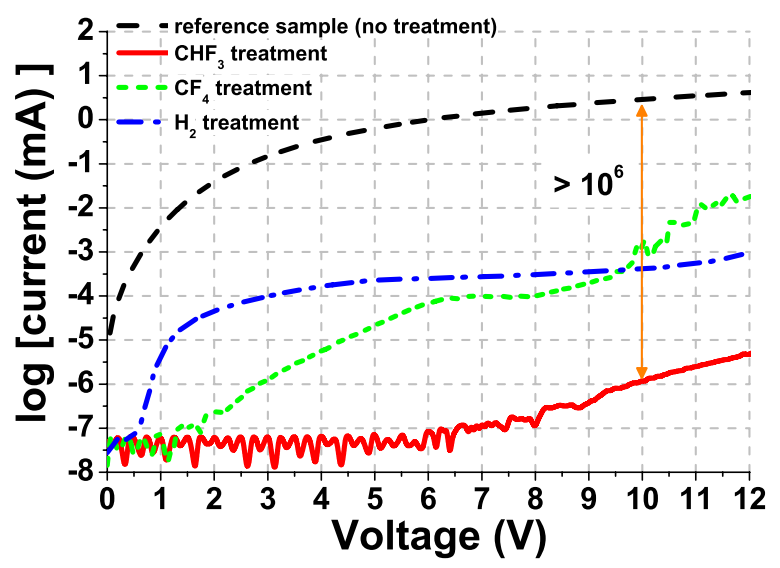

Figure 3. I-V characteristics of co-planar CTLM Ti/Au electrode structures deposited onto $\mathrm{p}-\mathrm{GaN}$ and having undergone different pre-metallization plasma treatments.

plasma treatments were applied to two more structures, and the results are included in figure 3 . In both cases, a significant reduction in current is observed compared to the untreated structure. However, the current blocking effect is significantly higher with the $\mathrm{CHF}_{3}$ plasma treatment than with either the $\mathrm{H}_{2}$ or the $\mathrm{CF}_{4}$ treatments alone, which shows that it is likely that both effects happen simultaneously. Relating the behavior just discussed to the operation of our light-structured LEDs, the low lateral diffusion length of carriers in p-GaN [17] confines light emission to an area directly below or close to the point of current injection on the $\mathrm{p}-\mathrm{GaN}$ surface. Consequently, one can easily understand that there is no light emission from the plasma treated area, in which vertical carrier injection is inhibited. As noted already, the semi-transparent $\mathrm{NiO}_{x}-\mathrm{Au}$ current spreading layer over the $\mathrm{p}-\mathrm{GaN}$ is not degraded by the plasma, and serves to electrically interconnect the untreated areas.

In order to illustrate the high resolution achievable by our technique, an array of disc emitters with decreasing nominal diameter down to $2 \mu \mathrm{m}$ (the resolution limit of our mask aligner) was transferred using the $\mathrm{CHF}_{3}$ plasma treatment onto a single LED. Figure 4(a) shows the line-scan of light intensity across the diameter of the smallest disc emitter as shown in the inset. This smallest emitter shows a full width at half maximum of $2.5 \mu \mathrm{m}$, demonstrating the capability of the $\mathrm{CHF}_{3}$ plasma treatment to fabricate elements comparable to the lithographic resolution limit. We anticipate that smaller emitters with submicron size could be fabricated with a higher resolution patterning technique such as e-beam, deep UV or nanoimprint lithography. Figure $4(b)$ shows the injection current versus forward bias voltage (I-V) characteristics for one LED before and after the image-transfer with the $\mathrm{CHF}_{3}$ plasma treatment. The turn-on voltage of the LED $(\sim 3 \mathrm{~V})$ does not change during the process, demonstrating that the junction properties are unaffected by the plasma treatment, consistent with the results discussed already indicating a shallow modification depth for the p-GaN. However, an increase in the differential resistance $r_{d}$ is clearly visible after the $\mathrm{CHF}_{3}$ treatment to create localized emitting regions (dashed curve in figure $4(b)$ ). As expected, the increase in $r_{d}$ after plasma treatment depends on the area ratio 

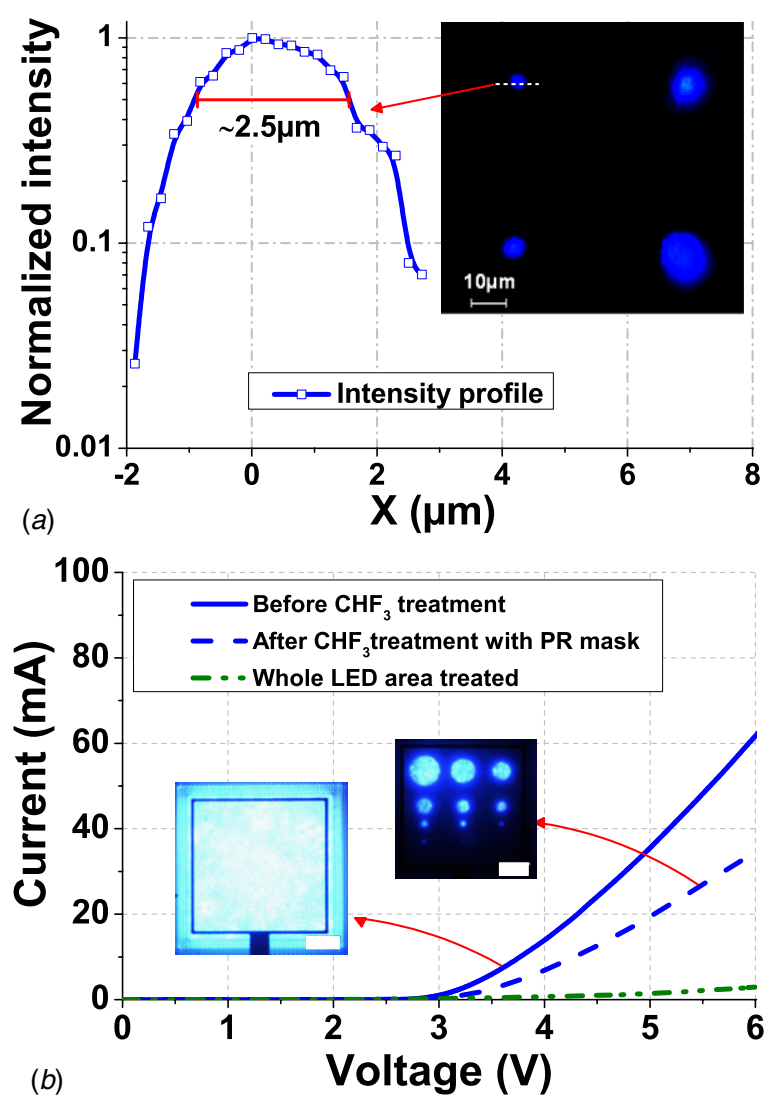

Figure 4. (a) Intensity profile across the diameter of the smallest emitting feature shown in the inset. The inset shows four microdisc-shape emitters with different diameters, fabricated by the $\mathrm{CHF}_{3}$ plasma treatment on a single mesa. (b) I-V characteristics before and after $\mathrm{CHF}_{3}$ plasma treatment of a single LED pixel. Emission pattern before and after $\mathrm{CHF}_{3}$ treatment is shown in the inserted images. No light can be detected when the whole LED is plasma treated. Scale bars (white) in both images represent $50 \mu \mathrm{m}$.

of exposed and mask-protected surface. The limiting case from a plasma treatment with no PR mask is shown in the figure; no visible light emission occurred although the current passed above turn-on is still detectable.

\subsection{Maskless photolithography}

Finally, the use of these light-structured LEDs was investigated for maskless photolithography, an application in which the compactness, high-brightness and high resolution of sources are key requirements. A $405 \mathrm{~nm}$ light-structured LED displaying $10 \mu \mathrm{m}$-wide bars separated by gaps of increasing width from $1 \mu \mathrm{m}$ up to $10 \mu \mathrm{m}$ (as shown in figure $1(b)$ ) was used to investigate the resolution limit of the pattern transfer from the light-structured LED to a PR-coated sample using a custom-made imaging setup described previously [5]. An LED was driven at an injection current of $60 \mathrm{~mA}$, providing an average optical power density of $\sim 1.3 \mathrm{~W} \mathrm{~cm}^{-2}$ on the test sample placed at the focal plane. The exposure time was set to $3 \mathrm{~s}$, giving an average exposure dose of $\sim 4 \mathrm{~J} \mathrm{~cm}^{-2}$ similar to the dose deduced from the manufacturer's datasheet. After exposure, the sample was soaked $30 \mathrm{~s}$ in developer to reveal the pattern transferred and dried under air. Due to the negativetone nature of the PR used, the area exposed to the LED light
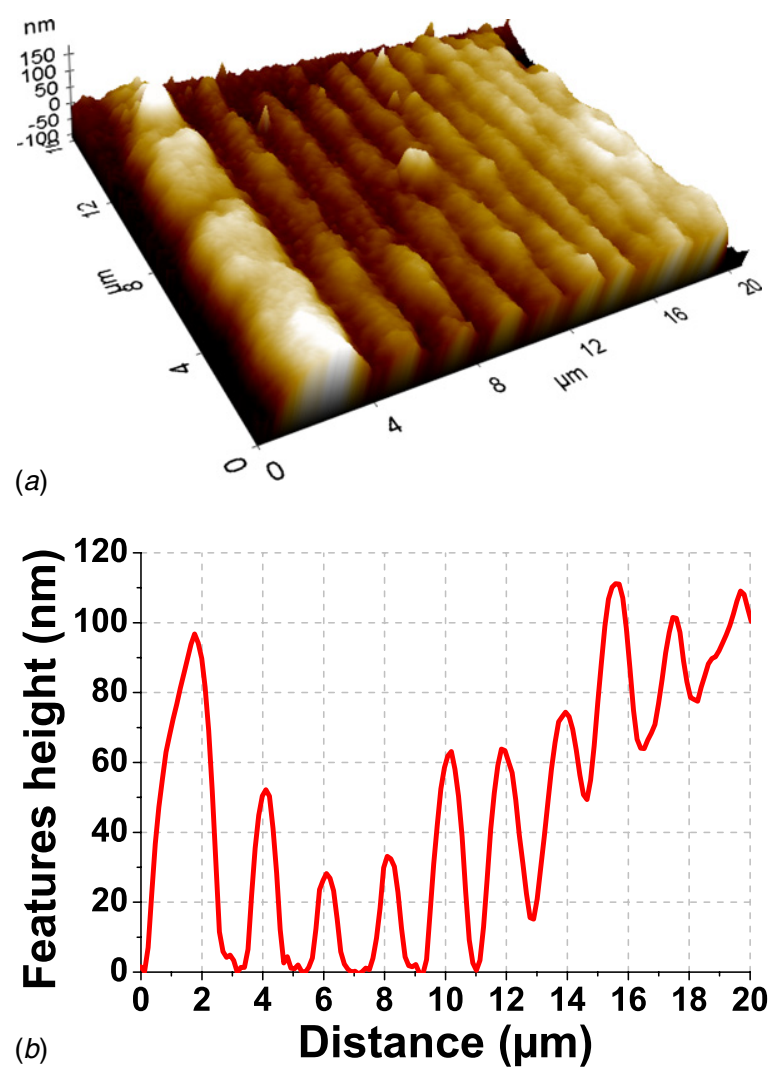

Figure 5. AFM image of the photoresist pattern made by maskless photolithography with a light-structured LED $(a)$ and typical height profile extracted from the same image along the perpendicular direction to the stripes $(b)$.

remains after development. The transferred pattern was then analyzed with an atomic force microscope (AFM) and a typical image is shown on figure 5(a). The extracted height profile is shown in figure $5(b)$. Due to the 10-to-1 demagnification, a perfect transfer would provide $1 \mu \mathrm{m}$-wide PR bars with intermediate gaps decreasing in width from $1 \mu \mathrm{m}$ down to $100 \mathrm{~nm}$. However because of the combined of effects of nonideality of the optical system, the divergence of the LED light emission and the diffraction limits, only the five largest gaps have been fully transferred into the PR layer, in the sense of the AFM probe reaching the Si substrate. The well-developed bars show a $\sim 1 \mu \mathrm{m}$-width at their base, as expected, with submicron gaps in-between down to $\sim 800 \mathrm{~nm}$. The non-uniform thickness of the PR features is attributed in part to imperfect uniformity of the LED emission, but such pattern definition is already good enough for further processing steps, for example pattern transfer by dry-etching into a harder mask layer such as $\mathrm{SiO}_{2}$ or metal. We anticipate that improved PR patterning could be obtained with further optimization of the imaging writing setup and process, as well as by using light-structured LEDs emitting at shorter wavelengths.

\section{Conclusions}

In summary, a new technique allowing the fabrication of single GaN-based LEDs with spatially microstructured light output patterns was demonstrated. This approach uses a 
$\mathrm{CHF}_{3}$-based plasma treatment at room temperature to modify the topmost p-GaN layer, leading to spatial modulation of current injection into the LED active region when some areas are protected from the plasma using a protecting patterned photoresist layer. Single devices fabricated in this manner can display images with micrometer resolution, while keeping electrical and optical performance similar to a unpatterned reference LEDs. Such a light-structured device emitting at $405 \mathrm{~nm}$ was used to demonstrate pattern transfer into PR by maskless photolithography. We anticipate that this new technique will be useful in a large range of applications with integrated optical sources, including micro-displays, bioinstrumentation, photolithography and lab-on-chip systems where compactness and low cost are of prime importance.

\section{Acknowledgments}

This work was supported by the UK EPSRC under the project EP/F05999X/1 'HYPIX'. Michael Wallace, Elaine Taylor and Paul Edwards from the University of Strathclyde department of Physics are acknowledged for their help in taking PL spectra at $325 \mathrm{~nm}$ excitation wavelength.

\section{Q2 References}

[1] Crawford M H 2009 LEDs for solid-state lighting: performance challenges and recent advances IEEE J. Select. Top. Quantum Electron. 15 1028-40

[2] Choi H W, Jeon C W and Dawson M D 2004 High-resolution $128 \times 96$ nitride microdisplay IEEE Electron. Device Lett. 25 277-9

[3] McKendry J D, Massoubre D, Zhang S, Rae B R, Green R P, Gu E, Henderson R K, Kelly A E and Dawson M D 2012 Visible-light communications using a CMOS-controlled micro-light-emitting-diode array IEEE J. Ligthwave Technol. 30 61-67

[4] Zarowna-Dabrowska A et al 2011 Miniaturized optoelectronic tweezers controlled by GaN micro-pixel light emitting diode arrays Opt. Express 19 2720-8

[5] Guilhabert B, Massoubre D, Richardson E, McKendry J J D, Valentine G, Henderson R K, Watson I M, Erdan Gu and Dawson M D 2012 Sub-micron lithography using
InGaN micro-lEDs: mask-free fabrication of LED arrays IEEE Photon. Technol. Lett. 24 2221-4

[6] Li Z L, Li K H and Choi H W 2010 Mechanism of optical degradation in microstructured InGaN light-emitting diodes J. Appl. Phys. 108114511

[7] Pearton S J, Zolper J C, Shul R J and Ren F 1999 Gan: processing, defects, and devices J. Appl. Phys. $861-78$

[8] Polyakov A Y, Smirnov N B, Govorkov A V, Pearton S J and Zavada J M 2003 Proton implantation effects on electrical and luminescent properties of p-GaN J. Appl. Phys. 94 3069-74

[9] Polyakov A Y, Smirnov N B, Govorkov A V, Baik K H, Pearton S J, Luo B, Ren F and Zavada J M 2003 Hydrogen plasma passivation effects on properties of p-GaN J. Appl. Phys. 94 3060-965

[10] Kuo T-W, Lin S-X, Hung P-K, Chong K-K, Hung C-I and Houng M-P 2010 Formation of selective high barrier region by inductively coupled plasma treatment on GaN-based light-emitting diodes Japan. J. Appl. Phys. 49116504

[11] Cai Y, Zhou Y, Chen K J and Lau K M 2005 High-performance enhancement-mode $\mathrm{AlGaN} / \mathrm{GaN}$ HEMTs using fluoride-based plasma treatment IEEE Electron. Device Lett. 26 435-7

[12] Liday J, Hotový I, Sitter H, Schmidegg K, Vogrinčič P, Bonnani A, Breza J, Ecke G and Vávra I 2007 Auger electron spectroscopy of $\mathrm{Au} / \mathrm{NiO}_{x}$ contacts on $\mathrm{p}-\mathrm{GaN}$ annealed in $\mathrm{N}_{2}$ and $\mathrm{O}_{2}+\mathrm{N}_{2}$ ambients Appl. Surf. Sci. 253 3174-80

[13] Elfström D et al 2006 Mask-less ultraviolet photolithography based on CMOS-driven micro-pixel light emitting diodes Opt. Express 17 23522-9

[14] Day J, Li J, Lie D Y C, Bradford C, Lin J Y and Jiang H X 2011 III-nitride full-scale high-resolution microdisplays Appl. Phys. Lett. 99031116

[15] Grieshaber W, Schubert E F, Goepfert I D, Karlicek R F, Schurman M J and Tran C 1996 Competition between band gap and yellow luminescence in $\mathrm{GaN}$ and its relevance for optoelectronic devices J. Appl. Phys. 80 4615-20

[16] Chu R, Suh C S, Wong M H, Fichtenbaum N, Brown D, McCarthy L, Keller S, Wu F, Speck J S and Mishra U K 2007 Impact of $\mathrm{CF}_{4}$ plasma treatment on GaN IEEE Electron. Device Lett. 28 781-3

[17] Hickmana R et al $2000 \mathrm{GaN}$ PN junction issues and developments Solid-State Electron. 44 377-81 


\section{QUERIES}

Page 1

Q1

Author: Please be aware that the color figures in this article will only appear in color in the Web version. If you require color in the printed journal and have not previously arranged it, please contact the Production Editor now.

\section{Page 6}

Q2

Author: Please check the details for any journal references that do not have a blue link as they may contain some incorrect information. Pale purple links are used for references to arXiv e-prints. 